\title{
Editorial
}

Nephrology

\section{Analgesic Polypharmacy Is a Likely Link to Increased Hospital Admissions}

\author{
Jeffrey Fudin ${ }^{a-d} \quad$ Amelia Persico $^{c}$ \\ ${ }^{a}$ Western New England University College of Pharmacy, Springfield, MA, USA; ${ }^{b}$ Albany College of Pharmacy \\ and Health Sciences, Albany, NY, USA; ' Stratton VA Medical Center, Albany, NY, USA; ${ }^{d}$ President, Remitigate \\ Therapeutics, Delmar, NY, USA
}

\section{Keywords \\ Opioids · Gabapentinoids · Dialysis · End-stage \\ renal disease $\cdot$ Chronic pain}

A recent study of 153,758 dialysis patients conducted by Waddy [1] found that $19 \%$ of the patients were concomitantly prescribed an opioid and a gabapentinoid. Their study sought to explore the relationship between gabapentinoid prescriptions, opioid prescriptions, and morbidity and mortality. In recent years, they note prescriptions for gabapentinoids have increased in opioid sparing efforts in the wake of the opioid epidemic [1]. At the same time, studies have found that gabapentinoids may serve to augment the "high" that opioid users obtain leading to increased abuse of gabapentinoids [2]. Further evidence has accumulated recently of patients with respiratory depression on gabapentinoid therapy [3]. This presents concerns for the risks of abuse and the risk of respiratory depression, particularly in patients with comorbid medical conditions such as end-stage renal disease (ESRD).

Chronic pain, both neuropathic and nociceptive, is a common ailment among ESRD and dialysis patients impacting up to $92 \%$ of this population [4]. Treating pain in this high-risk group requires detailed understanding of the pathophysiology of ESRD, the dialysis process as it impacts pharmacokinetics, and knowledge of pharmacology and pharmacokinetics of the commonly prescribed therapies [4]. Of particular importance is consideration of the reduced renal clearance of parent drugs and their active metabolites in between dialysis sessions coupled with the potentially enhanced clearance of these medications during dialysis. Decreased clearance increases the risk of toxicity.

Gabapentin and pregabalin are both renally eliminated unchanged in the urine and require dose adjustments for patients with ESRD and those on dialysis [4]. Population-based pharmacokinetic data have established that hemodialysis removes approximately $35 \%$ of gabapentin and $50-60 \%$ of pregabalin [5]. These impacts recommended dosing schemes as supplemental doses are generally recommended following hemodialysis [4]. This, in turn, has a significant impact on safety as patients who skip hemodialysis or are unable to tolerate a full hemodialysis session may be at risk for accumulation of these medications.

Gabapentinoids exert their efficacy in reducing neuropathic pain by binding to the alpha-2-delta subunit of $\mathrm{N}$-type voltage-gated calcium channels in the central nervous system (CNS) and reducing influx of calcium through the channels [4]. This subsequently reduces the outflow of excitatory neurotransmitters to mitigate neu-

\section{KARGER}

(c) 2020 S. Karger AG, Basel

karger@karger.com

www.karger.com/ajn
Jeffrey Fudin, BS, PharmD, FCCP, FASHP, FFSMB, DAIPM 
ropathic pain. It is well known that the combination of gabapentinoids, opioids, and other CNS depressants increases the risk of drowsiness, sedation, and confusion. These risks are compounded in elderly patients, patients with polypharmacy, and those with other medical comorbidities. The contribution of gabapentinoids to the risk of respiratory depression has yet to be thoroughly described in the literature. However, the timing of this study coincides with a warning from the US Food and Drug administration (FDA) regarding the risk of respiratory depression in patients who are prescribed gabapentinoids [3].

The statement from the FDA, released in late 2019, indicates that patients with preexisting respiratory risk factors such as concomitant opioid medications, other CNS depressants, advanced age, and pulmonary conditions are at increased risk of respiratory depression [3]. This warning comes following multiple cases reported in the FDA Adverse Event Reporting System (FAERS) database and review of medical literature. Within their statement, the FDA advises that this warning provides information contrary to widely held beliefs that gabapentinoids are without drug - drug interactions and have large therapeutic indices [3]. The present study by Waddy [1] serves to explore one subset of patients who could be at additional risk of respiratory depression when concomitantly prescribed opioids and gabapentinoids. The FDA does not state that patients on hemodialysis are one of the groups at increased risk. However, it raises questions regarding how a patient on hemodialysis fits into the picture.

Unfortunately, the present study fell short in its efforts to answer this question. Limitations of the study were not addressed by the authors, the breadth of which limits the applicability of their findings. First and foremost, this study did not adequately control for confounding variables including patients with polypharmacy and patients taking additional CNS depressants particularly those often prescribed in the pain space. Tricyclic antidepressants, SNRIs, SSRIS, benzodiazepines, and skeletal muscle relaxants are not mentioned, nor were the reasons for hospital admissions. The risk of concomitant benzodiazepine and opioid use is well-established in the literature yet not addressed here either [6]. Significant value would have been added to these findings if the daily dose of gabapentinoids prescribed was assessed. Including the number of tablets prescribed per month is not helpful as gabapentin, for example, is formulated in strengths ranging from 100 to $800 \mathrm{mg}$ per tablets and capsules. It would have been equally valuable to disclose the type and dose of opioid prescribed to evaluate respiratory risk, potential cytochrome (CYP) P450 interactions, and several other issues including but not limited to fall risk, endocrine disorders, and more.

Further exploration of potential drug-druginteractions is necessary. Although gabapentinoids are without CYP enzyme interactions, most commonly prescribed opioids have a multitude of CYP and p-glycoprotein interactions [7]. The findings of this study could suggest that tapentadol, an atypical opioid that undergoes phase II metabolism, has minimal CYP interactions, and no active metabolites is a safer option for patients on hemodialysis. It has also been posited that, despite a lack of pharmacokinetic interactions, gabapentinoids may be subject to pharmacodynamic interactions. For example, reduced gastrointestinal motility secondary to opioid therapy can increase AUC of gabapentinoids due to increased absorption [7].

The population studied by Waddy [1] was found to have lower risk of mortality when they were prescribed neither an opioid nor a gabapentinoid. This finding appears well aligned with their statement that there has been concern recently regarding unintended consequences of gabapentinoid prescriptions individually and in combination with opioids in dialysis patients. However, given the plethora of confounding variables, it is not possible to attribute this finding to any one variable. Perhaps this subset of the population was also less likely to be prescribed a benzodiazepine or had a lower burden of polypharmacy. The authors go on to state that patients who were prescribed an opioid and a gabapentinoid faced higher risk of hospitalization than those who were prescribed only opioids but did not face a higher mortality rate. They noted that patients with prior pain hospitalizations, women, those with cancer, those residing in nursing homes, and those living in poverty were more likely to have concomitant prescriptions for opioids and gabapentinoids [1]. This certainly begs the question as to whether or not these factors predisposed this subset of the population to a higher risk of hospitalization regardless of pharmacotherapy.

This study along with the recent warnings from the FDA set the stage for further exploration of the role concomitant use of gabapentinoids and opioids may play in respiratory depression, the opioid epidemic, and morbidity and mortality. However, more tightly controlled research is needed to support the types of conclusions proposed by Waddy [1] as many of their findings could be attributed to spurious correlation. There is simply not sufficient information to draw the blanket conclusion that opioids combined with gabapentinoids are un- 
safe with consideration of all these factors. Their conclusions suggest that a nonpharmacologic approach should be emphasized in patients with ESRD and that gabapentinoids may not be a safe option. Although there is a large body of evidence to support an interdisciplinary and multimodal approach to pain management for all patients, this study, in and of itself, does not provide significant evidence to support this conclusion. Findings of morbidity and mortality in this study cannot be confi- dently attributed to gabapentinoids, opioids, or the combination, as there were too many confounding variables.

\section{Disclosure Statement}

Drs. Amelia Persico and Jeffrey Fudin have no conflicts of interest to disclose.

\section{References}

1 Waddy SP. Concomitant use of gabapentinoids with opioids is associated with increased mortality and morbidity among dialysis patients. Am J Nephrol. 2020;DOI:10.1159/000507725.

2 Baird CR, Fox P, Colvin LA. Gabapentinoid abuse in order to potentiate the effect of methadone: a survey among substance misusers. Eur Addict Res. 2014;20(3):115-8.

3 U.S. Food and Drug Administration. FDA warns about serious breathing problems with seizure and nerve pain medicine gabapentin
(Neurontin, Gralise, Horizant) and pregabalin (Lyrica, Lyrica CR) when used with CNS depressants or in patients with lung problems. 2019.

4 Raouf M, Bettinger J, Wegrzyn EW, Mathew RO, Fudin JJ. Pharmacotherapeutic Management of Neuropathic Pain in End-Stage Renal Disease. Kidney Dis. 2020:1-1.

5 Raouf M, Atkinson TJ, Crumb MW, Fudin J. Rational dosing of gabapentin and pregabalin in chronic kidney disease. J Pain Res. 2017 Jan;10(Jan):275-8.
6 Gressler LE, Martin BC, Hudson TJ, Painter JT. Relationship between concomitant benzodiazepine-opioid use and adverse outcomes among US veterans. Pain. 2018 Mar;159(3): 451-9.

7 Feng XQ, Zhu LL, Zhou Q. Opioid analgesicsrelated pharmacokinetic drug interactions: from the perspectives of evidence based on randomized controlled trials and clinical risk management. J Pain Res. 2017 May; 10:122539. 